\title{
Therapeutic efficacy of lenvatinib for hepatocellular carcinoma with iso-high intensity in the hepatobiliary phase of Gd-EOB-DTPA-MRI
}

\author{
AKIFUMI KUWANO, KOSUKE TANAKA, MASAYOSHI YADA, SHIGEHIRO NAGASAWA, \\ YUSUKE MORITA, AKIHIDE MASUMOTO and KENTA MOTOMURA \\ Department of Hepatology, Iizuka Hospital, Iizuka, Fukuoka 820-8505, Japan
}

Received October 7, 2021; Accepted December 8, 2021

DOI: $10.3892 / \mathrm{mco} .2021 .2486$

\begin{abstract}
Previous studies have reported that hepatocellular carcinoma (HCC) harboring $\mathrm{WNT} / \beta$-catenin mutations exhibits iso-high intensity by gadolinium ethoxybenzyl diethylenetriaminepentaacetic acid-enhanced magnetic resonance imaging (Gd-EOB-DTPA-MRI, i.e. EOB-MRI) during the hepatobiliary phase (HBP), thus indicating that EOB-MRI may help clinicians identify an immune exclusion class, which might not respond to treatment with immune checkpoint inhibitors. The present study analyzed the efficacy of lenvatinib for HCC with iso-high intensity during the HBP of EOB-MRI. Overall, 52 patients who underwent EOB-MRI for $140 \mathrm{HCC}$ nodules were classified into iso-high-intensity and low-intensity groups during the HBP of EOB-MRI. The clinical and histological characteristics, and different responses to treatment of both groups were analyzed. The expression levels of $\beta$-catenin and glutamine synthetase, indicative of $\mathrm{WNT} / \beta$-catenin mutations, were enhanced in the HCC with iso-high-intensity group. Nine patients had iso-high intensity, whereas 43 patients
\end{abstract}

Correspondence to: Dr Kosuke Tanaka, Department of Hepatology, Iizuka Hospital, 3-83 Yoshio-machi, Iizuka, Fukuoka 820-8505, Japan

E-mail: ktanakah8@aih-net.com

Abbreviations: HCC, hepatocellular carcinoma; MTA, molecular targeted agent; ICI, immune checkpoint inhibitor; HBP, hepatobiliary phase; EOB-MRI, gadolinium ethoxybenzyl diethylenetriaminepentaacetic acid-enhanced magnetic resonance imaging; LEN, lenvatinib; CR, complete response; PR, partial response; $\mathrm{SD}$, stable disease; $\mathrm{PD}$, progressive disease; ORR, objective response rate; DCR, disease control rate; IHC, immunohistochemistry; GS, glutamine synthetase; CTNNB1, catenin $\beta-1$; OS, overall survival; PFS, progression-free survival; BCLC, Barcelona Clinic Liver Cancer; PIVKA-II, vitamin K absence or antagonist-II; OATP1B3, organic anion transporting polypeptide $1 \mathrm{~B} 3$; $\mathrm{CI}$, confidence interval

Key words: hepatocellular carcinoma, lenvatinib, WNT/ $\beta$-catenin mutations, hepatobiliary phase, Gd-EOB-DTPA-MRI had low intensity. Tumor size was larger, and the levels of antagonist-II or vitamin $\mathrm{K}$ absence were higher in the iso-high-intensity group. Furthermore, 3/9 patients in the iso-high-intensity group had objective responses compared with $13 / 43$ patients in the low-intensity group. Disease control was observed in $5 / 9$ patients in the iso-high-intensity group and 26/43 patients in the low-intensity group. Median overall survival was 29.8 months for the iso-high-intensity group compared with 20.8 months for the low-intensity group. In the iso-high-intensity group, the median progression-free survival rate was 6.7 months compared with 5.6 months in the low-intensity group. No differences in best percentage change from baseline tumor size were observed in either group. Although few patients were included in this study, the present findings suggested that the efficacy of lenvatinib was unaffected by signal intensity during the HBP of EOB-MRI.

\section{Introduction}

A previous study reported $85-90 \%$ of cases of primary liver cancer were hepatocellular carcinoma (HCC); globally, the third cause of death related to cancer (1). Recent advances in systemic chemotherapy for advanced HCC, including molecular targeted agents (MTAs) and immune checkpoint inhibitor (ICI) therapies, have improved patient prognosis; however, it is important to select agents appropriate for the personalized treatment of HCC (2-4).

Around $11-37 \%$ of $\mathrm{HCC}$ cases harbor mutations of $\mathrm{WNT} / \beta$-catenin that lead to the immune microenvironment lacking immune cell filtration, so called 'immune exclusion' or 'non-inflamed cold', in HCC (5). Furthermore, HCC with immune exclusion associated with mutations of $\mathrm{WNT} / \beta$-catenin is resistant to ICI therapies (6-8). Therefore, it is important to identify the subclass of HCC with or without immune exclusion induced by $\mathrm{WNT} / \beta$-catenin mutations before treatment. Recently, HCC treatment has focused on this subclass classification.

Ideally, mutations of WNT/ $\beta$-catenin would be identified using a non-invasive technique rather than by liver biopsy. Previous studies demonstrated that HCC harboring mutations of WNT/ $\beta$-catenin had iso-high intensity during the hepatobiliary phase (HBP) by magnetic resonance imaging 
enhanced by gadolinium ethoxybenzyl diethylenetriaminepentaacetic acid (Gd-EOB-DTPA-MRI, i.e. EOB-MRI) indicating EOB-MRI may help identify an immune exclusion class which might not respond to treatment with immune checkpoint inhibitors $(9,10)$. Around $12-22 \%$ of HCCs have iso-high intensity during the HBP of EOB-MRI $(11,12)$. EOB-MRI may be a non-invasive alternative to liver biopsy, which requires a histological method to identify mutations of Wnt/ $\beta$-catenin.

Previous studies have demonstrated how MTAs affect survival of advanced HCC patients (3). Lenvatinib (LEN) is an oral multikinase inhibitor of vascular endothelial growth factor (VEGF) receptors 1-3, fibroblast growth factor (FGF) receptors 1-4, platelet-derived growth factor (PDGF) receptor $\alpha, \operatorname{RET}$, and $\operatorname{KIT}(13,14)$. An international randomized, open-label trial that was performed in multiple centers to investigate the non-inferiority of sorafenib (REFLECT; NCT01761266) reported LEN significantly improved progression-free survival (PFS) compared with sorafenib in patients with previously untreated, metastatic, or unresectable HCC. LEN has been approved for HCC treatment (3).

The therapeutic efficacy of LEN for HCC with mutations of $\mathrm{WNT} / \beta$-catenin has not been reported. Here, we analyzed LEN efficacy for HCC that may have $\mathrm{WNT} / \beta$-catenin mutations with iso-high intensity during the HBP of EOB-MRI.

\section{Materials and methods}

Patients. This prospective single-center study analyzed LEN efficacy for HCC that may have $\mathrm{WNT} / \beta$-catenin mutations with iso-high intensity during the HBP of EOB-MRI. Eighty-one patients received LEN for non-resectable HCC at Iizuka Hospital from May 2018 to February 2021. We excluded 26 patients who did not undergo EOB-MRI within 3 months prior to LEN administration and three patients who were observed for $<12$ weeks. We evaluated 52 patients by EOB-MRI and of these, we enrolled 140 HCC (Fig. 1). This study was performed according to the Declaration of Helsinki guidelines and was approved by Iizuka Hospital ethics committee (approval no. 18070). All patients gave written informed consent.

Treatment protocol. Patients received doses of LEN according to body weight ( $8 \mathrm{mg}$ /day for bodyweight $<60 \mathrm{~kg}$, or $12 \mathrm{mg} /$ day for bodyweight $\geq 60 \mathrm{~kg}$ ) (Eisai Co., Ltd.). Dose interruption and potential subsequent reduction (to $8 \mathrm{mg} / \mathrm{day}, 4 \mathrm{mg} /$ day, or $4 \mathrm{mg}$ every other day) were permitted when patients developed LEN-related adverse events. The protocols in the REFLECT trial were prescribed by Eisai Co., Ltd. (3). Common Terminology Criteria for Adverse Events, version 4.0 was used to grade adverse events. Adverse events $\geq$ grade 3 or any unacceptable grade 2 adverse events were a cause for reduced drug dose or interrupted treatment as per guidelines for LEN administration. Following occurrence of an adverse event, LEN dose was reduced or temporarily interrupted until symptoms were grade 1 or 2, as per the Eisai Co., Ltd. guidelines.

Evaluation of treatment efficacy. Physicians evaluated antitumor responses using modified RECIST v.1.1 (15). The disease control rate (DCR) was determined according to complete response (CR), partial response (PR), or stable disease (SD) present for $\geq 4$ months. The objective response rate (ORR) was defined as PR or CR. Each nodule was evaluated according to the top three sized nodules in each case.

$E O B-M R I$. Characterization and pretreatment staging for $\mathrm{HCC}$ was determined by Gd MRI using a 1.5-T or 3.0-T MR system (Ingeina; Philips Healthcare) using the same protocol. For the dynamic study, $0.1 \mathrm{ml}$ Primovist $(0.25 \mathrm{mmol} / \mathrm{ml}$ gadoxetic acid; Bayer Schering Pharma) was administered intravenously per $\mathrm{kg}$ /bodyweight. The optimal arterial dominant phase, calculated as time of peak enhancement in the abdominal aorta + an additional $10 \mathrm{sec}$ of imaging time (16-22 sec) was achieved using the test injection method with $1.5 \mathrm{ml}$ gadoxetic acid $+8-\mathrm{ml}$ saline flush. Subsequent to imaging during the arterial phase, portal and equilibrium phase images were captured at 20 and $60 \mathrm{sec}$, respectively, once the previous imaging phase was over. The HBP for all patients was obtained 20 min post-injection.

Imaging analysis. Four hepatologists performed imaging analysis in a blind fashion without clinicopathological information. Qualitative analysis of HCC with iso-high intensity during the HBP of EOB-MRI was defined by a signal intensity higher than the pre-contrast image.

Immunohistochemical (IHC) analyses. Liver tumor biopsy samples fixed with $10 \%$ formalin were paraffin embedded. Serial sections $(5-\mu \mathrm{m})$ were sectioned from paraffin blocks of liver tissues and stained with hematoxylin-eosin. The presence of glutamine synthetase (GS) and $\beta$-catenin was determined in 14 HCC specimens by IHC with the following primary antibodies: monoclonal mouse anti-human $\beta$-catenin (\#610153; BD Biosciences; 1/300 dilution) or monoclonal mouse anti-human GS (\#GS-6; Millipore; 1/500 dilution). A Bond Polymer System was used to develop reactions (Leica Biosystems) related to secondary antibodies. $\beta$-catenin staining in the nucleus is indicative of an activating mutation in the catenin $\beta-1$ (CTNNB1) gene $(16,17)$ and strong GS diffuse staining is indicative of the constitutive activation of WNT/ $\beta$-catenin signaling associated with $\beta$-catenin mutations (18). Therefore, the presence of $\beta$-catenin nuclear staining in $\geq 5 \%$ of tumor cells (19) or strong diffuse GS staining were considered to demonstrate the activation of $\mathrm{WNT} / \beta$-catenin signaling (9).

Statistical analysis. JMP Pro Version 11 statistical software was used for all analyses (SAS Institute, Inc.). Results were shown as the median (inter-quartile range). Significant differences between groups were examined by $\chi^{2}$, Fisher's exact or Mann-Whitney U test. The Kaplan-Meier technique was used for the statistical analyses of overall survival (OS) and PFS; significant differences in OS and PFS were determined by log-rank analysis. Statistical significance was determined when $\mathrm{P}<0.05$.

\section{Results}

Subjects' characteristics. Patient characteristics are shown in Table I. The iso-high-intensity group contained patients 


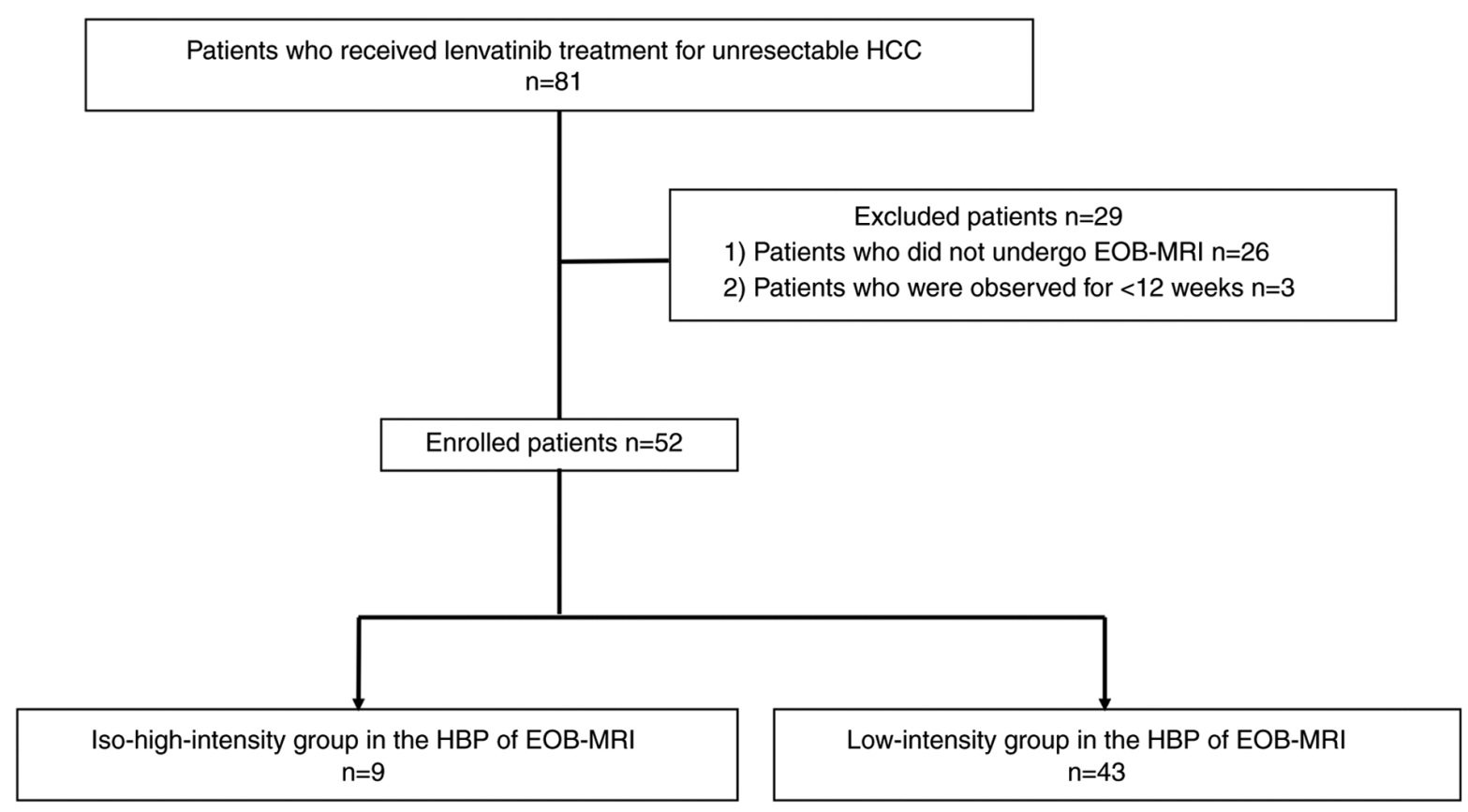

Figure 1. Patient flow chart. HCC, hepatocellular carcinoma; EOB-MRI, gadolinium ethoxybenzyl diethylenetriaminepentaacetic acid-enhanced magnetic resonance imaging; HBP, hepatobiliary phase.

with iso-high-intensity and low-intensity nodules. There were nine patients $(17.3 \%)$ and 14 HCC nodules $(10 \%)$ with iso-high intensity and 43 patients $(82.7 \%)$ and $126 \mathrm{HCC}$ nodules $(90.0 \%)$ with low intensity. Five patients $(55.6 \%)$ had both iso-high-intensity and low-intensity nodules in the iso-high-intensity group. Tumor size was larger in the iso-high-intensity group vs the low-intensity group $[6.2 \mathrm{~cm}$ (3.25-8.35) vs. $2.5 \mathrm{~cm}$ (1.6-3.9), $\mathrm{P}=0.004]$. The levels of antagonist-II (PIVKA-II) or vitamin K absence were higher in the iso-high-intensity group [ $938 \mathrm{mAU} / \mathrm{ml}(26.5-31832.5)$ vs. $140 \mathrm{mAU} / \mathrm{ml}$ (32-18112), $\mathrm{P}=0.0137]$. There were two patients with Barcelona Clinic Liver Cancer (BCLC) stage A, four with stage $\mathrm{B}$, and three with stage $\mathrm{C}$ in the iso-high-intensity group. In addition, there were five patients with BCLC stage $A, 23$ with stage $B$ and 15 with stage $C$ in the low-intensity group. Age, sex, etiology, Child-Pugh grade, microvascular invasion (MVI), extrahepatic spread (HIS), and serum $\alpha$-fetoprotein levels were similar between groups.

IHC of $\beta$-catenin and GS in HCC tissues. The expressions of $\beta$-catenin and GS in 13 patients (four patients in the iso-high-intensity group and nine patients in the low-intensity group) were assessed by IHC before LEN therapy. Typical magnetic resonance images and immunohistochemical findings are presented in Fig. 2A and B. All four patients $(100 \%)$ were positive for $\beta$-catenin or GS staining in the iso-high-intensity group, and 3/9 (33.3\%) were positive for $\beta$-catenin or GS staining in the low-intensity group (Table II); however, there were no differences in $\beta$-catenin $(\mathrm{P}=1.00)$ and GS $(\mathrm{P}=0.07)$ staining between the two groups because of the small patient numbers in these groups.

Efficacy of LEN in the iso-high intensity and low-intensity groups. The OS, PFS, ORR, and DCR were similar between groups. The ORR (CR+PR) was 3/9 (33.3\%) in the iso-high-intensity group and 13/43 (30.2\%) in the low-intensity group $(\mathrm{P}=0.62)$. The DCR $(\mathrm{CR}+\mathrm{PR}+\mathrm{SD})$ was $5 / 9(55.6 \%)$ in the iso-high intensity group and 26/43 (60.5\%) in the low-intensity group $(\mathrm{P}=0.45)$ (Table III). Median OS was 29.8 months [95\% confidence interval $(\mathrm{CI})=7.1-31.3$ ] in the iso-high-intensity group versus 20.7 months $(95 \% \mathrm{CI}=11.2-26.3)$ in the low-intensity group $(\mathrm{P}=0.84)$. The median $\mathrm{PFS}$ was 6.7 months (95\% CI $=2.9-11.9$ ) in the iso-high-intensity group compared with 5.6 months $(95 \% \mathrm{CI}=3.7-7.9)$ in the low-intensity group ( $\mathrm{P}=0.95$; Fig. 3).

We also evaluated the change in each HCC tumor treated with LEN. The best percentage change was $-10.5 \%$ in the iso-high-intensity group $(4.99 \pm 0.44 \mathrm{~cm}$ to $4.79 \pm 0.54 \mathrm{~cm})$ and $1.1 \%$ in the low-intensity group $(1.88 \pm 0.15 \mathrm{~cm}$ to $1.88 \pm 0.18 \mathrm{~cm}$; Fig. 4). We compared the values of the best percentage change between groups using Mann-Whitney U test. The best percentage change from the baseline tumor size $(\mathrm{P}=0.67)$, the ORR [28.6\% (4/14) in the iso-high-intensity group and $27.8 \%(35 / 126)$ in the low-intensity group $(\mathrm{P}=0.73)]$, and the DCR [92.9\% (13/14) in the iso-high-intensity group and 73.0\% $(92 / 126)$ in the low-intensity group $(\mathrm{P}=0.39$; Table IV)] were similar between groups.

\section{Discussion}

Our current study suggested that the status of mutations of WNT/ $\beta$-catenin may not influence LEN effectiveness. Approximately $30 \%$ of $\mathrm{HCC}$ cases were reported to show the constitutive activation of WNT/ $\beta$-catenin signaling, induced by the relevant gene mutations (20). The negative influence of WNT/ $\beta$-catenin activation on the DCR and PFS of patients receiving anti-PD-1 therapy $(7,8,21)$. However, the PFS was similar between sorafenib-treated patients with and without mutations in WNT/ $\beta$-catenin genes who had undergone gene sequencing (10). Although the efficacy of sorafenib and other 
Table I. Characteristics of patients in the iso-high-intensity and low-intensity groups of HBP in EOB-MRI.

\begin{tabular}{|c|c|c|c|c|}
\hline Characteristic & All & Iso-high-intensity group & Low-intensity group & P-value \\
\hline Number & 52 & 9 & 43 & \\
\hline Age, years & $73(68-79.75)$ & $82(75-87)$ & $73(68-78)$ & 0.686 \\
\hline $\operatorname{Sex}, \mathrm{M} / \mathrm{F}$ & $36 / 16$ & $7 / 2$ & $29 / 14$ & 0.53 \\
\hline Etiology & & & & 0.3 \\
\hline $\mathrm{HCV}$ & $23(44.2 \%)$ & $1(11.1 \%)$ & $22(51.2 \%)$ & \\
\hline $\mathrm{HBV}$ & $10(19.2 \%)$ & $2(22.2 \%)$ & $8(18.6 \%)$ & \\
\hline Alcohol & $9(17.3 \%)$ & $2(22.2 \%)$ & $7(16.3 \%)$ & \\
\hline $\mathrm{AIH} / \mathrm{PBC}$ & $2(3.8 \%)$ & $0(0 \%)$ & $2(4.7 \%)$ & \\
\hline Cryptogenic & $8(15.5 \%)$ & $4(44.5 \%)$ & $4(9.2 \%)$ & \\
\hline Max tumor size, $\mathrm{cm}$ & $2.7(1.7-4.9)$ & $6.2(3.25-8.35)$ & $2.5(1.6-3.9)$ & 0.0004 \\
\hline Size of intrahepatic lesion $>3 \mathrm{~cm}$ & $24(46.2 \%)$ & $7(77.8 \%)$ & $17(39.5 \%)$ & 0.0364 \\
\hline Number of intrahepatic lesions $>5$ & $31(59.6 \%)$ & $5(55.6 \%)$ & $26(60.4 \%)$ & 0.7849 \\
\hline MVI positive & $11(21.2 \%)$ & $1(11.1 \%)$ & $10(23.3 \%)$ & 0.69 \\
\hline EHS positive & $12(23.1 \%)$ & $1(11.1 \%)$ & $11(25.6 \%)$ & 0.29 \\
\hline Child-Pugh score & & & & 0.63 \\
\hline $5 \mathrm{~A}$ & $29(55.8 \%)$ & $6(72.7 \%)$ & $23(53.5 \%)$ & \\
\hline $6 \mathrm{~A}$ & $13(25 \%)$ & $1(11.1 \%)$ & $12(27.9 \%)$ & \\
\hline$>7$ & $10(19.2 \%)$ & $2(22.2 \%)$ & $8(18.6 \%)$ & \\
\hline Albumin & $3.65(3.3-4.1)$ & $3.7(3.4-4.1)$ & $3.6(3.3-4.2)$ & 0.79 \\
\hline Total bilirubin & $0.8(0.6-1.2)$ & $1.1(0.8-2.3)$ & $0.8(0.6-1.1)$ & 0.0068 \\
\hline BCLC & & & & 0.118 \\
\hline A & $7(13.5 \%)$ & $2(22.2 \%)$ & $5(11.6 \%)$ & \\
\hline $\mathrm{B}$ & $27(51.9 \%)$ & $4(44.4 \%)$ & $23(53.5 \%)$ & \\
\hline C D & $18(34.6 \%)$ & $3(33.4 \%)$ & $15(34.9 \%)$ & \\
\hline \multicolumn{5}{|l|}{ Tumor marker } \\
\hline $\operatorname{AFP}(\mathrm{ng} / \mathrm{ml})$ & $10.1(3.58-188.88)$ & $6.3(3.3-6054.8)$ & $10.3(4.1-201.8)$ & 0.692 \\
\hline PIVKA-II (mAU/ml) & $181.5(32-3390)$ & $938(26.5-31832.5)$ & $140(32-18112)$ & 0.0137 \\
\hline LEN dose & & & & 0.5604 \\
\hline $12 \mathrm{mg}$ & $4(7.7 \%)$ & $0(0 \%)$ & $4(9.3 \%)$ & \\
\hline $8 \mathrm{mg}$ & $27(51.9 \%)$ & $4(44.4 \%)$ & $23(53.4 \%)$ & \\
\hline $4 \mathrm{mg}$ & $21(40.4 \%)$ & $5(54.6 \%)$ & $16(37.2 \%)$ & \\
\hline
\end{tabular}

Data are expressed as the median and interquartile range, or as numbers and percentages (\%). EOB-MRI, gadolinium ethoxybenzyl diethylenetriaminepentaacetic acid-enhanced magnetic resonance imaging; HCV, hepatitis C; HBV, hepatitis B; AIH, autoimmune hepatitis; PBC, primary biliary cholangitis; MVI, macroscopic portal vein invasion; EHS, extrahepatic spread; BCLC, Barcelona Clinic Liver Cancer; AFP, $\alpha$-fetoprotein; PIVKA-II, vitamin K absence or antagonist-II; LEN, lenvatinib.

MTAs might be independent of $\mathrm{WNT} / \beta$-catenin mutation status, clinical evidence demonstrating how responses of HCC patients to LEN are affected by WNT/ $\beta$-catenin activation and how an immune cold phenotype is acquired, is still lacking.

$\mathrm{WNT} / \beta$-catenin pathway activation promotes $\beta$-catenin accumulation in the cytoplasm, its nuclear translocation, and diffuse accumulation of GS, a transcriptional target of $\beta$-catenin (22-24). The presence of WNT/ $\beta$-catenin mutations is determined by IHC analysis showing the nuclear expression of $\beta$-catenin or cytoplasmic overexpression of GS in HCC tissues, indicating they might be useful biomarkers of $\mathrm{WNT} / \beta$-catenin mutations (22-24).
Furthermore, GS and $\beta$-catenin expressions were reported to correlate with OATP1B3 expression (25) and HCC with WNT/ $\beta$-catenin mutations showed iso-high intensity during the HBP of EOB-MRI $(9,10)$. The mechanism involves the induction of OATP1B3 (an EOB transporter) by WNT/ $\beta$-catenin mutations $(9,26)$. Therefore, iso-high intensity during the HBP of EOB-MRI might be useful as an imaging biomarker of $\mathrm{HCC}$ patients with mutations of $\mathrm{WNT} / \beta$-catenin. The frequency of HCCs with iso-high intensity was $12-22 \%$ during the HBP of EOB-MRI $(11,12)$. We investigated the immunostaining of $\beta$-catenin and GS for the detection of mutations of WNT/ $\beta$-catenin. Several HCC tissues with iso-high intensity during the HBP of EOB-MRI showed GS or $\beta$-catenin positive 
A

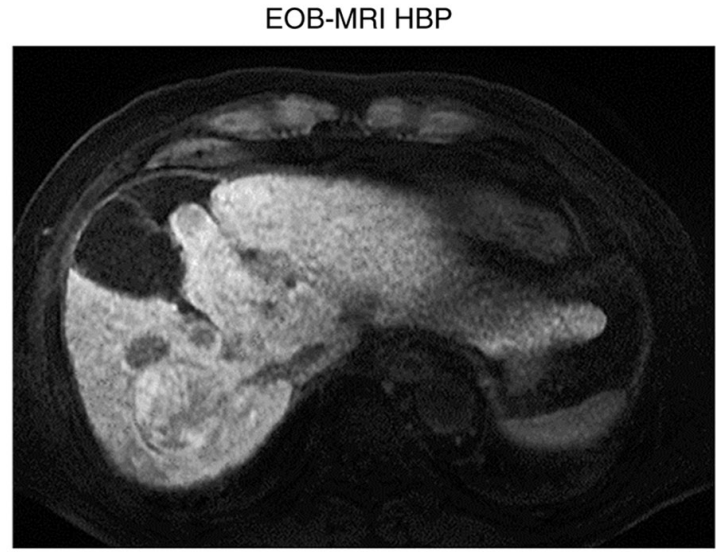

$\beta$-catenin

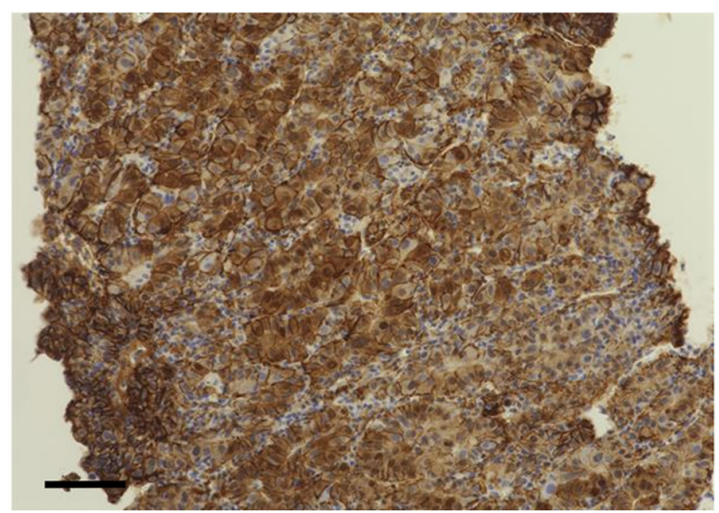

B

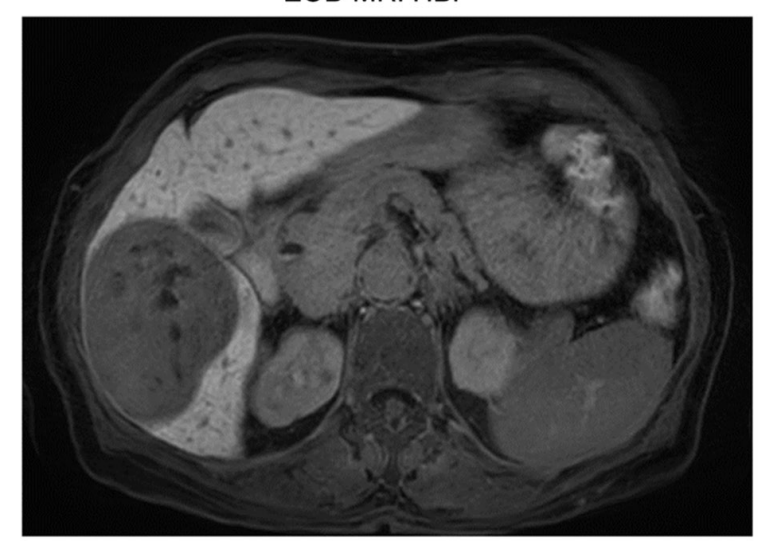

$\beta$-catenin

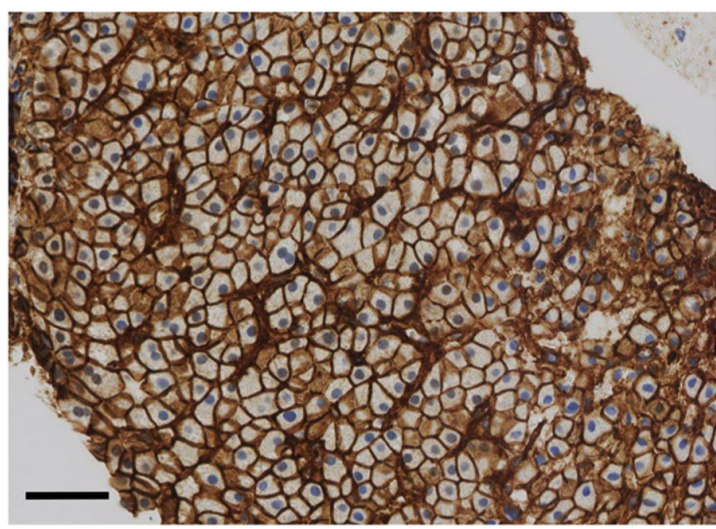

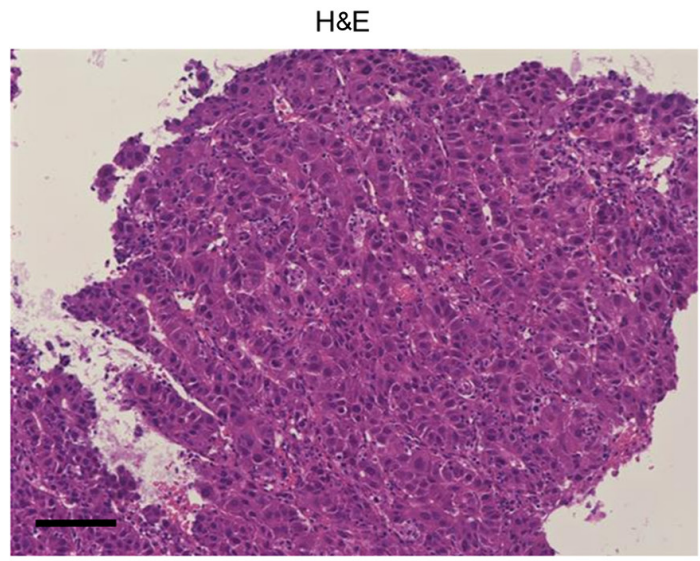

GS

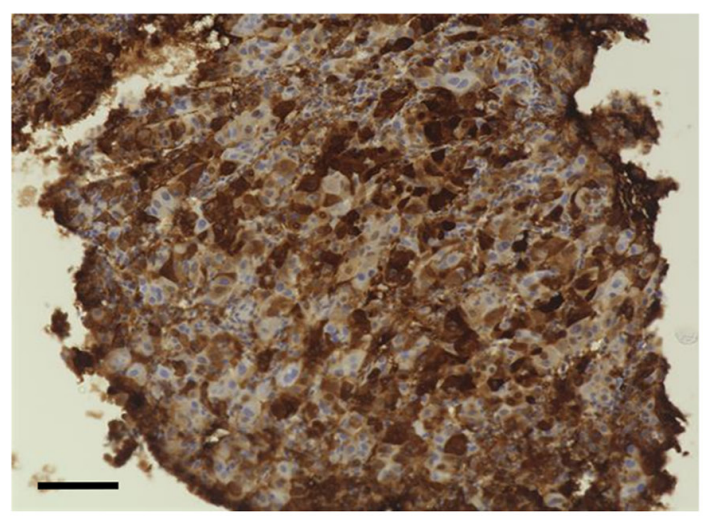

H\&E

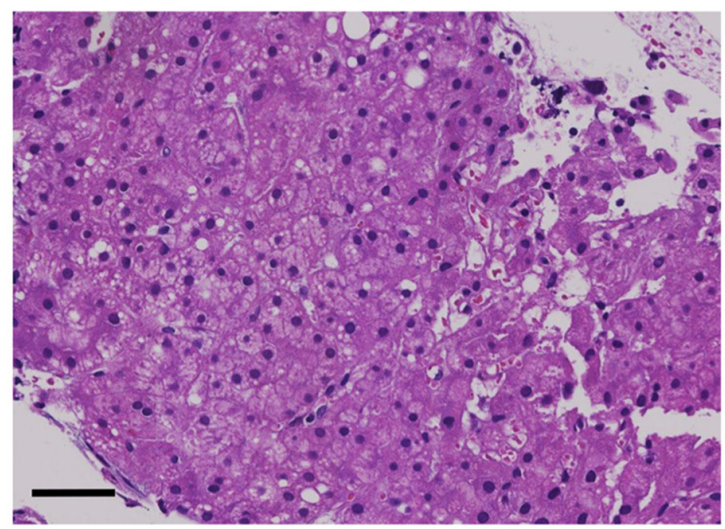

GS

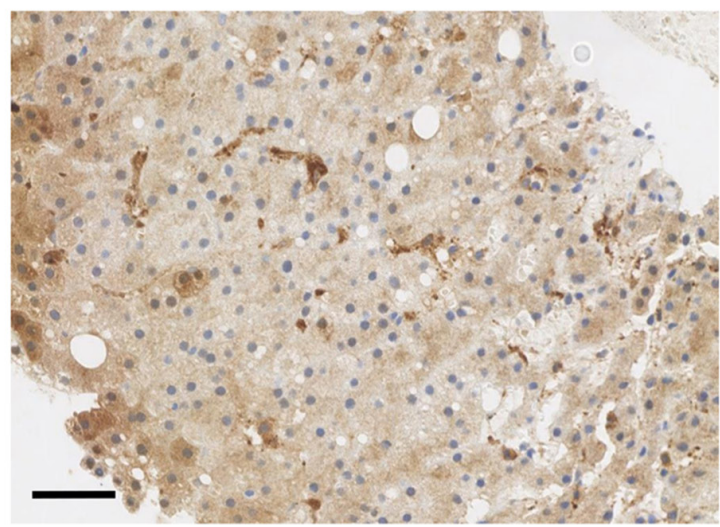

Figure 2. Typical MR images and immunohistochemical findings. (A) HCC with iso-high-intensity in the HBP of EOB-MRI. (B) HCC with the low-intensity in the HBP of EOB-MRI. H\&E staining of liver sections (magnification, x200; black scale bar, $100 \mu \mathrm{m}$ ). $\beta$-catenin and GS staining of liver tissue was performed to evaluate Wnt/ $\beta$-catenin mutations (magnification, x200; black scale bar, $100 \mu \mathrm{m}$ ). EOB-MRI, gadolinium ethoxybenzyl diethylenetriaminepentaacetic acid-enhanced magnetic resonance imaging; GS, glutamine synthetase; HBP, hepatobiliary phase; H\&E, hematoxylin and eosin. 
Table II. Relationship between Wnt/ $\beta$-catenin mutations and signal intensity in the HBP of EOB-MRI in 13 patients.

\begin{tabular}{rlcclc}
\hline Case & Group & Age, years & Sex & Etiology & $\beta$-catenin \\
\hline 1 & iso-high & 82 & M & ALC & Positive \\
2 & iso-high & 58 & F & HBV & Negative \\
3 & iso-high & 83 & P & NBNC & Negative \\
4 & iso-high & 87 & M & NBNC & Positive \\
5 & Low & 68 & P & HBV & Negative \\
6 & Low & 80 & P & AIH & Positive \\
7 & Low & 75 & M & HBV & Positive \\
8 & Low & 59 & M & ALC & Negative \\
9 & Low & 52 & HBV & Positive \\
10 & Low & 76 & P & HCV & Negative \\
11 & Low & 71 & HCV & Negative \\
13 & Low & 78 & P & HCV & Negative \\
\hline
\end{tabular}

HBP, hepatobiliary phase; EOB-MRI, gadolinium ethoxybenzyl diethylenetriaminepentaacetic acid-enhanced magnetic resonance imaging; ALC, alcohol; GS, glutamine synthetase.

Table III. Comparison of the response to lenvatinib between the EOB iso-high-intensity group and low-intensity group (patient analysis).

\begin{tabular}{lcc}
\hline LEN effectiveness & $\begin{array}{c}\text { Iso-high-intensity } \\
\text { group, } \mathrm{n}=9(\%)\end{array}$ & $\begin{array}{c}\text { Low-intensity } \\
\text { group, } \mathrm{n}=43(\%)\end{array}$ \\
\hline Overall response & $0(0)$ & \\
CR & $3(33.3)$ & $11(25.6)$ \\
PR & $2(22.3)$ & $13(30.2)$ \\
SD & $4(44.4)$ & $17(39.5)$ \\
PD & $3(33.3)$ & $13(30.2)$ \\
ORR (CR+PR) & $5(55.6)$ & $26(60.5)$ \\
DCR (CR+PR+SD) & & 0.14 \\
\hline
\end{tabular}

EOB, gadolinium ethoxybenzyl diethylenetriaminepentaacetic acid; LEN, lenvatinib; CR, complete response; PR, partial response; SD, stable disease; PD, progression disease; DCR, disease control rate; ORR, objective response rate.

staining. Thus, we confirmed mutations of WNT/ $\beta$-catenin in HCC with iso-high intensity during the HBP of EOB-MRI as previously reported (25).

Our findings suggested that the efficacy of LEN did not differ between the iso-high-intensity group, in which patients may have $\mathrm{WNT} / \beta$-catenin mutations, and the low-intensity group.

Clinical trials or studies using pre-clinical models to investigate immunomodulatory influences of antiangiogenic agents on the tumor microenvironment reported enhanced maturation of dendritic cells, trafficking and function of $\mathrm{T}$ cells, and reversed immunosuppression induced by hypoxia or immunosuppressive cells (27-29). Other in vivo and in vitro studies reported sorafenib enhanced antitumor immunity by promoting tumor-associated macrophage polarization to an M1 phenotype (30-32), enhancing the infiltration and functions of $\mathrm{CD}^{+}$and $\mathrm{CD}^{+} \mathrm{T}$ cells $(33,34)$, lowering numbers of Tregs (35-37), and reversing suppressive functions of myeloid-derived cells in tumor microenvironments $(22,38,39)$. Other MTAs including LEN were shown to promote antitumor immune activity in pre-clinical models (40-42). Many of these immunomodulatory effects of MTAs might be associated with the inhibition of VEGFR signaling (27). Compared with earlier tyrosine kinase inhibitors, LEN has a greater inhibitory effect on FGFR4 (43). WNT/ $\beta$-catenin mutations are associated with FGFR4 overexpression (43) suggesting the therapeutic effects of MTAs including LEN might not be affected by WNT/ $\beta$-catenin mutations although they might alter the 'non-inflamed cold' subclass to the 'inflamed hot' subclass in the immune microenvironment of $\mathrm{WNT} / \beta$-catenin-mutant HCC.

Fujita et al classified HCCs into one of the three groups on the basis of intensity during the HBP of EOB-MRI: i) homogeneous hypointensity; ii) heterogeneous hyperintensity; 


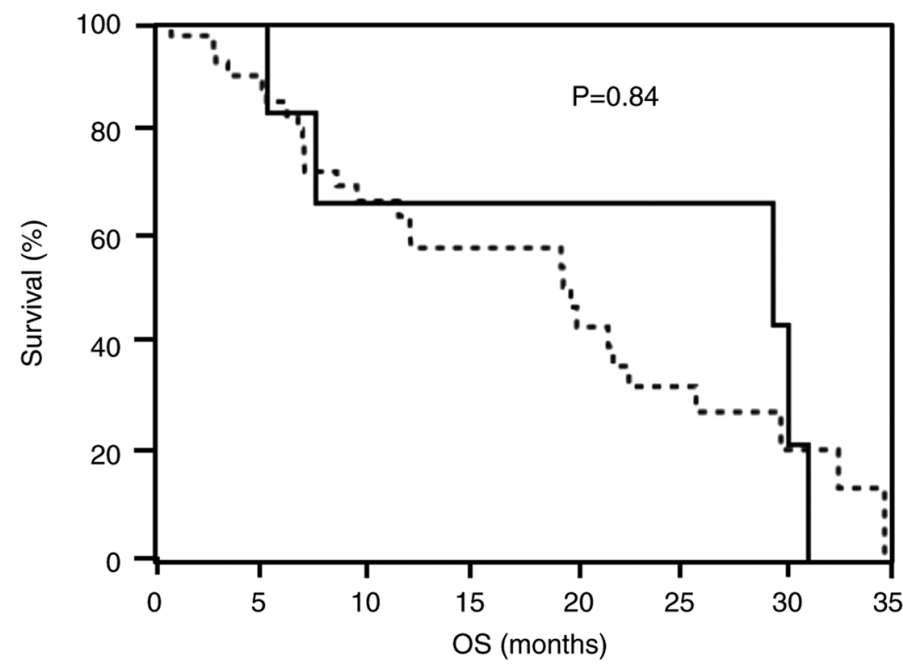

$\begin{array}{rcccccccr}\text { Iso-high-intensity group } & 9 & 9 & 5 & 4 & 4 & 4 & 3 & 1 \\ \text { Low-intensity group } & 43 & 39 & 29 & 20 & 17 & 9 & 5 & 1\end{array}$

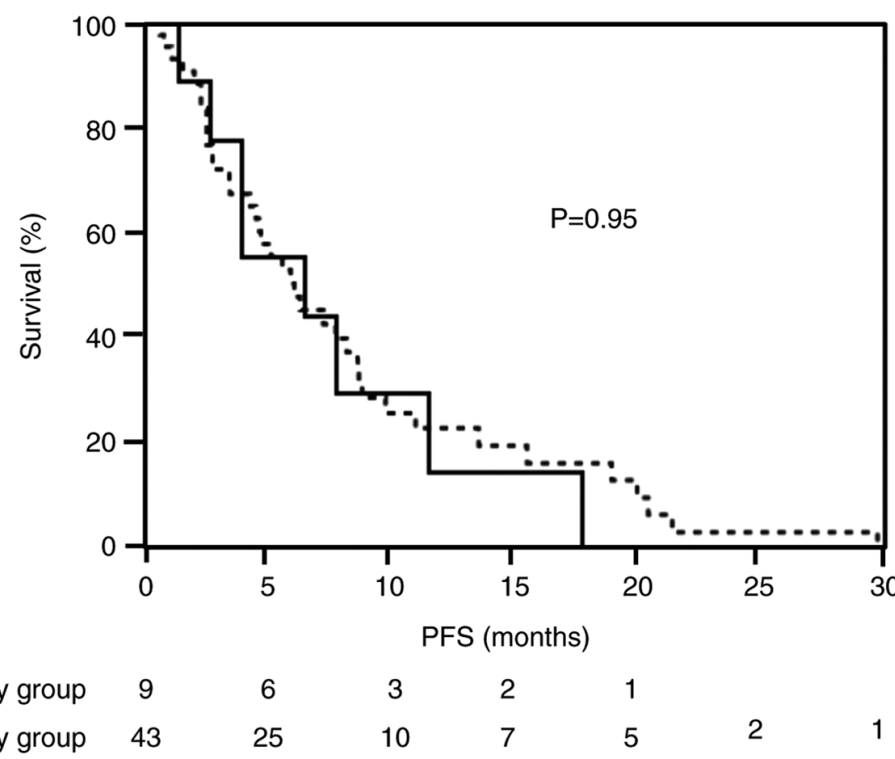

Figure 3. Kaplan-Meier estimates of OS and PFS in iso-high-intensity and low-intensity groups during HBP. Significant differences in OS and PFS were determined by log-rank analysis. OS, overall survival; PFS, progression-free survival; HBP, hepatobiliary phase.

and iii) homogeneous hyperintensity (44). During the HBP, all HCCs in the iso-high-intensity group in our study displayed heterogeneous hyperintensity (group 2). The tumor size was larger and PIVKA-II levels were higher in the iso-high-intensity group compared with the low-intensity group, similar to our previous study. Other studies reported that GS-positive HCCs were larger although PIVKA-II levels were not associated to tumor size. Therefore, PIVKA-II levels in the iso-high-intensity group of GS-positive HCCs might be higher than those in the low-intensity group $(45,46)$. Fujita et al recorded a disease-free survival rate in group 2 that was significantly lower compared with group 1 (44), but our study revealed the OS, PFS, ORR, and DCR were similar in all groups after LEN treatment.

Study limitations were as follows. First, this study was performed at a single center and therefore had limited numbers of HCC cases. Second, it was unclear whether the mutations of one tumor reflected the mutations in other masses when considering the heterogeneity of HCC in multiple masses. Third, we could not evaluate the frequency of $\beta$-catenin mutations in HCC with iso-high intensity or low-intensity during the HBP of EOB-MRI because biopsies were not obtained from all patients. We also had to consider that HCC with low intensity could also be positive for $\beta$-catenin or GS.

The results suggested that the efficacy of LEN was similar between the iso-high-intensity and low-intensity groups. Given these limitations, further studies enrolling a larger number of cases is needed before MTAs such as LEN can be used as a first-line treatment for HCC with iso-high intensity instead of ICIs alone or in combination with angiogenesis inhibitors, which are the most common first-line regimens for advanced HCC in many countries (2). These findings might allow us to suggest the early therapeutic evaluation of ICIs alone or in combination with angiogenesis inhibitors for HCC with iso-high intensity and quickly change to MTAs if the 
Table IV. Comparison of the response to lenvatinib between the EOB iso-high-intensity group and low-intensity group (nodule analysis).

\begin{tabular}{lccr}
\hline LEN effectiveness & $\begin{array}{c}\text { Iso-high-intensity } \\
\text { group, } \mathrm{n}=14(\%)\end{array}$ & $\begin{array}{c}\text { Low-intensity } \\
\text { group, } \mathrm{n}=126(\%)\end{array}$ & P-value \\
\hline Overall response & & & 0.85 \\
CR & $0(0)$ & $16(12.7)$ & \\
PR & $4(28.6)$ & $21(16.7)$ & \\
SD & $9(64.3)$ & $32(25.4)$ & 0.73 \\
PD & $1(7.1)$ & $35(27.8)$ & 0.39 \\
ORR (CR+PR) & $4(28.6)$ & $92(73.0)$ & \\
DCR (CR+PR+SD) & $13(92.9)$ & & \\
\hline
\end{tabular}

EOB, gadolinium ethoxybenzyl diethylenetriaminepentaacetic acid; LEN, lenvatinib; CR, complete response; PR, partial response; SD, stable disease; PD, progression disease; ORR, objective response rate; DCR, disease control rate.

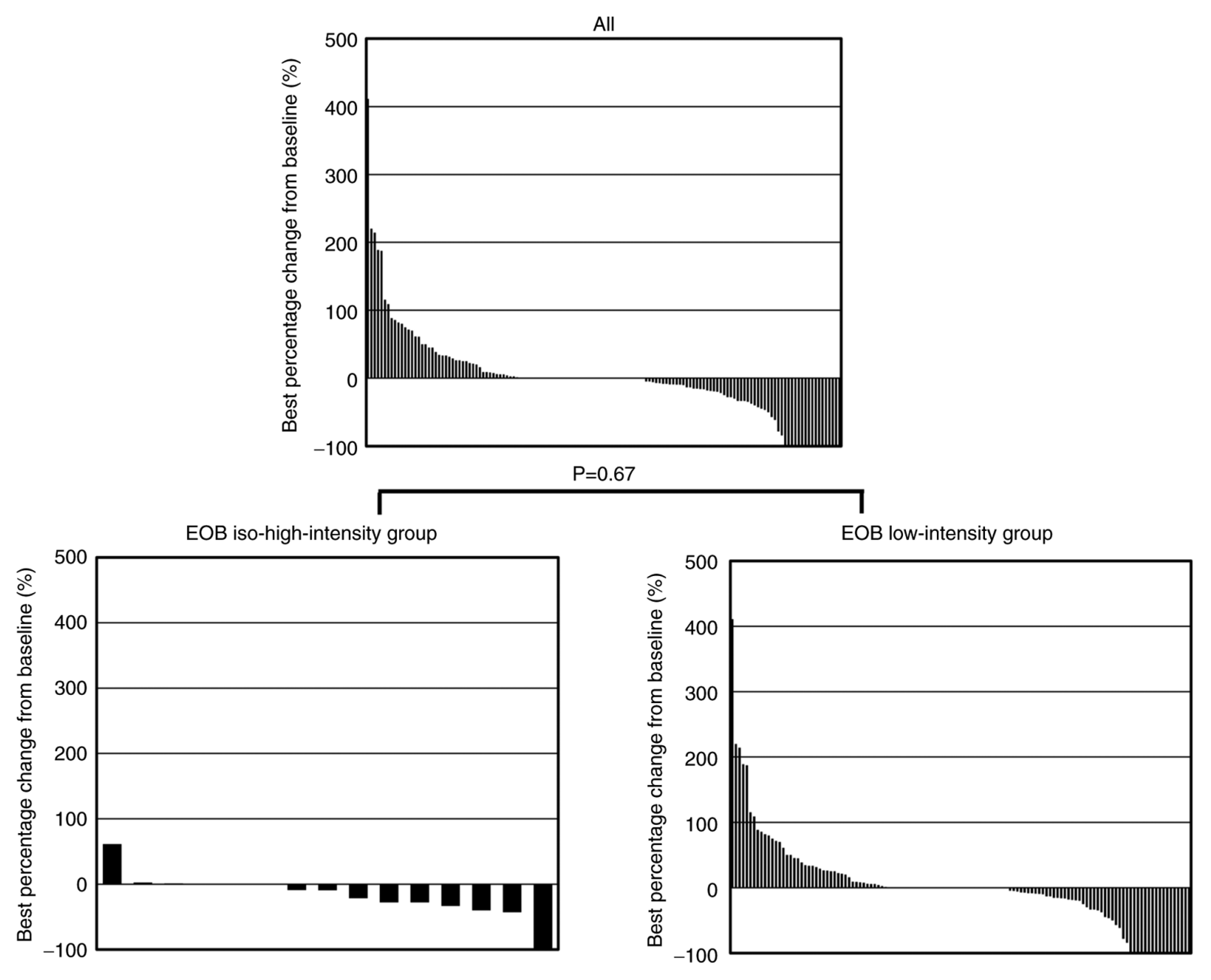

Figure 4. Best percentage change in iso-high-intensity and low-intensity groups during HBP. Significant differences in the values of the best percentage change between groups were examined by Mann-Whitney U-test. HBP, hepatobiliary phase.

initial treatment is ineffective. The findings of this study might allow the personalized treatment of patients with HCC by aiding the selection of appropriate therapeutic agents. However, these findings should be confirmed using a larger number of patients.

\section{Acknowledgements}

The authors would like to acknowledge Mrs. Y. Ishibashi (Department of Hepatology, Iizuka Hospital) for aiding preparation of the text. 


\section{Funding}

No funding was received.

\section{Availability of data and materials}

The datasets used and/or analyzed during the current study are available from the corresponding author on reasonable request.

\section{Authors' contributions}

AK, MY, KT, AM and KM designed the study. AK, MY, SN, $\mathrm{KT}$ and YM assisted with the data analyses. AK wrote the initial draft of the manuscript. MY and KM contributed to data analysis and interpretation. MY and KM helped prepare the manuscript and critically reviewed the manuscript. AK and KT confirm the authenticity of all the raw data. All authors read and approved the final manuscript.

\section{Ethics approval and consent to participate}

The research and protocol were in accord with the Declaration of Helsinki principles and the ethical guidelines of the 1975 Declaration of Helsinki, respectively. This study received approval from the Iizuka Hospital Ethics Committee (no. 18070). Written informed consent was obtained from all individual participants included in the study.

\section{Patient consent for publication}

Not applicable.

\section{Competing interests}

All authors declare that they have no competing interests.

\section{References}

1. Caldwell S and Park SH: The epidemiology of hepatocellular cancer: From the perspectives of public health problem to tumor biology. J Gastroenterol 44 Suppl: S96-S101, 2009.

2. Finn RS, Qin S, Ikeda M, Galle PR, Ducreux M, Kim TY, Kudo M, Breder V, Merle P, Kaseb AO, et al: Atezolizumab plus bevacizumab in unresectable hepatocellular carcinoma. N Engl J Med 382: 1894-1905, 2020

3. Kudo M, Finn RS, Qin S, Han KH, Ikeda K, Piscaglia F, Baron A, Park JW, Han G, Jassem J, et al: Lenvatinib versus sorafenib in first-line treatment of patients with unresectable hepatocellular carcinoma: A randomised phase 3 non-inferiority trial. Lancet 391: 1163-1173, 2018.

4. Eso Y and Marusawa H: Novel approaches for molecular targeted therapy against hepatocellular carcinoma. Hepatol Res 48: 597-607, 2018

5. Torbenson MS, Ng IO, Park YN, Roncalli M and Sakamoto M: Digestive system tumours: WHO classification of tumours. In: Hepatocellular Carcinoma. 5th edition. WHO Classification of Tumours Editorial Board. WHO Press, Geneva, pp229-239, 2019.

6. Sia D, Jiao Y,Martinez-Quetglas I,Kuchuk O, Villacorta-Martin C, Castro de Moura M, Putra J, Camprecios G, Bassaganyas L, Akers $\mathrm{N}$, et al: Identification of an immune-specific class of hepatocellular carcinoma, based on molecular features. Gastroenterology 153: 812-826, 2017.

7. Llovet JM, Montal R, Sia D and Finn RS: Molecular therapies and precision medicine for hepatocellular carcinoma. Nat Rev Clin Oncol 15: 599-616, 2018.
8. Pinyol R, Sia D and Llovet JM: Immune exclusion-Wnt/CTNNB1 class predicts resistance to immunotherapies in HCC. Clin Cancer Res 25: 2021-2023, 2019.

9. Ueno A, Masugi Y, Yamazaki K, Komuta M, Effendi K, Tanami Y, Tsujikawa H, Tanimoto A, Okuda S, Itano O, et al: OATP1B3 expression is strongly associated with $\mathrm{Wnt} / \beta$-catenin signalling and represents the transporter of gadoxetic acid in hepatocellular carcinoma. J Hepatol 61: 1080-1087, 2014.

10. Kudo M: Gd-EOB-DTPA-MRI could predict WNT/ $\beta$-catenin mutation and resistance to immune checkpoint inhibitor therapy in hepatocellular carcinoma. Liver Cancer 9: 479-490, 2020.

11. Tsuboyama T, Onishi H, Kim T, Akita H, Hori M, Tatsumi M, Nakamoto A, Nagano H, Matsuura N, Wakasa K and Tomoda K: Hepatocellular carcinoma: Hepatocyte-selective enhancement at gadoxetic acid-enhanced MR imaging-correlation with expression of sinusoidal and canalicular transporters and bile accumulation. Radiology 255: 824-833, 2010.

12. Kitao A, Matsui O, Yoneda N, Kozaka K, Kobayashi S, Koda W, Gabata T, Yamashita T, Kaneko S, Nakanuma Y, et al: Hypervascular hepatocellular carcinoma: Correlation between biologic features and signal intensity on gadoxetic acid-enhanced MR images. Radiology 265: 780-789, 2012.

13. Tohyama O, Matsui J, Kodama K, Hata-Sugi N, Kimura T, Okamoto K, Minoshima Y, Iwata M and Funahashi Y: Antitumor activity of lenvatinib (e7080): An angiogenesis inhibitor that targets multiple receptor tyrosine kinases in preclinical human thyroid cancer models. J Thyroid Res 2014: 638747, 2014.

14. Yamamoto Y, Matsui J, Matsushima T, Obaishi H, Miyazaki K, Nakamura K, Tohyama O, Semba T, Yamaguchi A, Hoshi SS, et al: Lenvatinib, an angiogenesis inhibitor targeting VEGFR/FGFR, shows broad antitumor activity in human tumor xenograft models associated with microvessel density and pericyte coverage. Vasc Cell 6: 18, 2014.

15. Lencioni R and Llovet JM: Modified RECIST (mRECIST) assessment for hepatocellular carcinoma. Semin Liver Dis 30: 52-60, 2010.

16. Hsu HC, Jeng YM, Mao TL, Chu JS, Lai PL and Peng SY: Beta-catenin mutations are associated with a subset of low-stage hepatocellular carcinoma negative for hepatitis B virus and with favorable prognosis. Am J Pathol 157: 763-770, 2000.

17. Laurent-Puig P, Legoix P, Bluteau O, Belghiti J, Franco D, Binot F, Monges G, Thomas G, Bioulac-Sage P and Zucman-Rossi J: Genetic alterations associated with hepatocellular carcinomas define distinct pathways of hepatocarcinogenesis. Gastroenterology 120: 1763-1773, 2001.

18. Zucman-Rossi J, Benhamouche S, Godard C, Boyault S, Grimber G, Balabaud C, Cunha AS, Bioulac-Sage P and Perret C: Differential effects of inactivated Axin1 and activated beta-catenin mutations in human hepatocellular carcinomas. Oncogene 26: 774-780, 2007.

19. Tsujikawa H, Masugi Y, Yamazaki K, Itano O, Kitagawa Y and Sakamoto M: Immunohistochemical molecular analysis indicates hepatocellular carcinoma subgroups that reflect tumor aggressiveness. Hum Pathol 50: 24-33, 2016.

20. Lee HC, Kim M and Wands JR: Wnt/Frizzled signaling in hepatocellular carcinoma. Front Biosci 11: 1901-1915, 2006.

21. Harding JJ, Nandakumar S, Armenia J, Khalil DN, Albano M, Ly M, Shia J, Hechtman JF, Kundra R, El Dika I, et al: Prospective Genotyping of hepatocellular carcinoma: Clinical implications of next-generation sequencing for matching patients to targeted and immune therapies. Clin Can Res 25: 2116-2126, 2019.

22. Nhieu JT, Renard CA, Wei Y, Cherqui D, Zafrani ES and Buendia MA: Nuclear accumulation of mutated beta-catenin in hepatocellular carcinoma is associated with increased cell proliferation. Am J Pathol 155: 703-710, 1999.

23. Cadoret A, Ovejero C, Terris B, Souil E, Lévy L, Lamers WH, Kitajewski J, Kahn A and Perret C: New targets of beta-catenin signaling in the liver are involved in the glutamine metabolism. Oncogene 21: 8293-8301, 2002.

24. Loeppen S, Schneider D, Gaunitz F, Gebhardt R, Kurek R, Buchmann A and Schwarz M: Overexpression of glutamine synthetase is associated with beta-catenin-mutations in mouse liver tumors during promotion of hepatocarcinogenesis by phenobarbital. Cancer Res 62: 5685-5688, 2002.

25. Kitao A, Matsui O, Yoneda N, Kozaka K, Kobayashi S, Sanada J, Koda W, Minami T, Inoue D, Yoshida K, et al: Hepatocellular carcinoma with $\beta$-catenin mutation: Imaging and pathologic characteristics. Radiology 275: 708-717, 2015.

26. Giles RH, van Es JH and Clevers H: Caught up in a Wnt storm: Wnt signaling in cancer. Biochim Biophys Acta 1653: 1-24, 2003. 
27. Ramjiawan RR, Griffioen AW and Duda DG: Anti-angiogenesis for cancer revisited: Is there a role for combinations with immunotherapy? Angiogenesis 20: 185-204, 2017.

28. Hegde PS, Wallin JJ and Mancao C: Predictive markers of anti-VEGF and emerging role of angiogenesis inhibitors as immunotherapeutics. Semin Cancer Biol 52: 117-124, 2018.

29. Kwilas AR, Donahue RN, Tsang KY and Hodge JW: Immune consequences of tyrosine kinase inhibitors that synergize with cancer immunotherapy. Cancer Cell Microenviron 2: e677, 2015.

30. Wang DY, Johnson DB and Davis EJ: Toxicities associated with PD-1/PD-L1 blockade. Cancer J 24: 36-40, 2018.

31. Sprinzl MF, Reisinger F, Puschnik A, Ringelhan M, Ackermann K, Hartmann D, Schiemann M, Weinmann A, Galle PR, Schuchmann M, et al: Sorafenib perpetuates cellular anticancer effector functions by modulating the crosstalk between macrophages and natural killer cells. Hepatology 57: 2358-2368, 2013 .

32. Wei X, Tang C, Lu X, Liu R, Zhou M, He D, Zheng D, Sun C and Wu Z: MiR-101 targets DUSP1 to regulate the TGF- $\beta$ secretion in sorafenib inhibits macrophage-induced growth of hepatocarcinoma. Oncotarget 6: 18389-18405, 2015.

33. Farsaci B, Donahue RN, Coplin MA, Grenga I, Lepone LM, Molinolo AA and Hodge JW: Immune consequences of decreasing tumor vasculature with antiangiogenic tyrosine kinase inhibitors in combination with therapeutic vaccines. Cancer Immunol Res 2: 1090-1102, 2014.

34. Romero AI, Chaput N, Poirier-Colame V, Rusakiewicz S, Jacquelot N, Chaba K, Mortier E, Jacques Y, Caillat-Zucman S, Flament C, et al: Regulation of CD4(+)NKG2D(+) Th1 cells in patients with metastatic melanoma treated with sorafenib: Role of IL-15R $\alpha$ and NKG2D triggering. Cancer Res 74: 68-80, 2014.

35. Sunay MM, Foote JB, Leatherman JM, Edwards JP, Armstrong TD, Nirschl CJ, Hicks J and Emens LA: Sorafenib combined with HER-2 targeted vaccination can promote effective T cell immunity in vivo. Int Immunopharmacol 46: 112-123, 2017.

36. Chuang HY, Chang YF, Liu RS and Hwang JJ: Serial low doses of sorafenib enhance therapeutic efficacy of adoptive T cell therapy in a murine model by improving tumor microenvironment. PLOS One 9: e109992, 2014.

37. Chen ML, Yan BS, Lu WC, Chen MH, Yu SL, Yang PC and Cheng AL: Sorafenib relieves cell-intrinsic and cell-extrinsic inhibitions of effector T cells in tumor microenvironment to augment antitumor immunity. Int J Cancer 134: 319-331, 2014.

38. Cabrera R, Ararat M, Xu Y, Brusko T, Wasserfall C, Atkinson MA, Chang LJ, Liu C and Nelson DR: Immune modulation of effector CD4+ and regulatory $T$ cell function by sorafenib in patients with hepatocellular carcinoma. Cancer Immunol Immunother 62 737-746, 2013
39. Chang CJ, Yang YH, Chiu CJ, Lu LC, Liao CC, Liang CW, Hsu CH and Cheng AL: Targeting tumor-infiltrating Ly $6 \mathrm{G}^{+}$ myeloid cells improves sorafenib efficacy in mouse orthotopic hepatocellular carcinoma. Int J Cancer 142: 1878-1889, 2018.

40. Kwilas AR, Ardiani A, Donahue RN, Aftab DT and Hodge JW Dual effects of a targeted small-molecule inhibitor (cabozantinib) on immune-mediated killing of tumor cells and immune tumor microenvironment permissiveness when combined with a cancer vaccine. J Transl Med 12: 294, 2014.

41. Tsai AK, Khan AY, Worgo CE, Wang LL, Liang Y and Davila E: A multikinase and DNA-PK inhibitor combination immunomodulates melanomas, suppresses tumor progression, and enhances immunotherapies. Cancer Immunol Res 5: 790-803, 2017.

42. Heine A, Schilling J, Grünwald B, Krüger A, Gevensleben H, Held SA, Garbi N, Kurts C, Brossart P, Knolle P, et al: The induction of human myeloid derived suppressor cells through hepatic stellate cells is dose-dependently inhibited by the tyrosine kinase inhibitors nilotinib, dasatinib and sorafenib, but not sunitinib. Cancer Immunol Immunother 65: 273-282, 2016.

43. Yamauchi M, Ono A, Ishikawa A, Kodama K, Uchikawa S, Hatooka H, Zhang P, Teraoka Y, Morio K, Fujino H, et al: Tumor fibroblast growth factor receptor 4 level predicts the efficacy of lenvatinib in patients with advanced hepatocellular carcinoma. Clin Transl Gastroenterol 11: e00179, 2020

44. Fujita N, Nishie A, Kubo Y, Asayama Y, Ushijima Y, Takayama Y, Moirta K, Shirabe K, Aishima S and Honda H: Hepatocellular carcinoma: Clinical significance of signal heterogeneity in the hepatobiliary phase of gadoxetic acid-enhanced MR imaging. Eur Radiol 25: 211-220, 2015.

45. Dal Bello B, Rosa L, Campanini N, Tinelli C, Torello Viera F, D'Ambrosio G, Rossi S and Silini EM: Glutamine synthetase immunostaining correlates with pathologic features of hepatocellular carcinoma and better survival after radiofrequency thermal ablation. Clin Cancer Res 16: 2157-2166, 2010.

46. Weitz IC and Liebman HA: Des-gamma-carboxy (abnormal) prothrombin and hepatocellular carcinoma: A critical review. Hepatology 18: 990-997, 1993.

This work is licensed under a Creative Commons Attribution-NonCommercial-NoDerivatives 4.0 International (CC BY-NC-ND 4.0) License. 